\title{
Prevalence and Antibiotic Susceptibility Pattern of Methicillin Resistant Staphylococcus aureus (MRSA) at a Tertiary Care Hospital from Northern India
}

\author{
Ayesha Nazar*, Yusuf Imran, Vichal Rastogi and Parul Singhal \\ Department of Microbiology, School of Medical Science and Research, \\ Sharda Hospital, Greater Noida, Uttar Pradesh, India
}

*Corresponding author

Keywords

World Health Organization (WHO), medicines, MRSA infections

Article Info

Accepted:

22 August 2019

Available Online:

10 September 2019

\section{A B S T R A C T}

MRSA is one of the major public health problems especially in developing countries where irrational use of antibiotics has led to greater levels of resistance. Increasing burden of MRSA infections in healthcare as well as community setting warrants constant surveillance. This study was conducted from January 2018 to June 2019 at a tertiary care hospital to know the prevalence and susceptibility pattern of MRSA. MRSA identification was done with cefoxitin disc diffusion method. Out of the total of 158 Staphylococcus aureus isolates, 59 (37.3\%) were MRSA and the remaining $99(62.6 \%)$ isolates were MSSA. In our study $47.4 \%$ of the MRSA isolates were hospital acquired MRSA while $52.5 \%$ were community acquired MRSA. Majority of the isolates were obtained from skin and soft tissue infections. This study showed a higher prevalence of CA-MRSA. Consistent surveillance of strains circulating in particular communities and hospitals along with formulation of rational antibiotic policy would be helpful in reducing the incidence of MRSA infections.

\section{Introduction}

Antimicrobial resistance is an important concern for the public health authorities at global level. It is one of the major public health problems especially in developing countries where relatively easy availability and higher consumption of medicines have led to disproportionately higher incidence of inappropriate use of antibiotics and greater levels of resistance compared to developed countries.

The CDC declared in 2013 that the human race is now in the "post-antibiotic era," and in 2014, the World Health Organization (WHO) warned that the antibiotic resistance crisis is becoming dire (WHO 2014). According to 
CDC estimates, 80461 invasive MRSA infections and 11285 MRSA related deaths occurred in 2011.

Methicillin resistant Staphylococcus aureus (MRSA) was first detected in Britain in 1961 and is now quite common in hospitals around the world. MRSA is now endemic in India. The incidence of MRSA varies from 25 per cent in western part of India to 50 per cent in South India (Patel AK 2010, Gopalakrishnan R 2010). A study conducted at 15 tertiary care centers in India by Indian Network for Surveillance of Antimicrobial Resistance (INSAR) group reported MRSA rates among outpatients, non-ICU inpatients and ICU patients as $28 \%, 42 \%$ and $43 \%$ respectively in 2008 and $27 \%, 49 \%$ and $47 \%$ respectively in 2009 (Joshi S. et 2013). Since the beginning of the MRSA expansion, infections due to this organism were primarily limited to major hospital centers and their healthcare systems. Community-acquired MRSA was rarely reported. However, during the 1990s, a new epidemic of MRSA began where MRSA strains were also isolated from apparently healthy individuals in the communities with no previous contact with healthcare facilities.CA-MRSA strains often express lower levels of resistance to oxacillin and multiply faster than HA-MRSA strains with significantly shorter doubling times which may help CA-MRSA achieve successful colonization by enabling it to out compete commensal bacterial flora. CA-MRSA strains are usually susceptible to multiple non- $\beta$ lactam antibiotics like trimethoprimsulfamethoxazole, clindamycin, tetracycline in contrast to the multidrug resistance usually seen in HA-MRSA strains (Robert $\mathrm{C}$ et al., 2012).

The $\beta$-lactam resistance of MRSA is caused by the acquisition of the exogenous mec $\mathrm{A}$ gene. The mecA gene complex is comprised of $m e c \mathrm{~A}$ together with its regulator genes, mecl and mec $\mathrm{R}$ which reside within a mobile genomic island known as the staphylococcal cassette chromosome mec (SCCmec). $\mathrm{SCC} m e c$ is composed of mec gene complex which confers resistance to methicillin, and the $c c r$ gene complex which encodes recombinases responsible for its mobility. (Deresinski S. et al., 2005). To date 13 SCCmec types have been discovered. (Lakhundi S.et al.,2018). The mecA gene encodes for an alternative penicillin-binding protein (PBP) designated PBP 2' (or PBP 2a). This altered PBP (i.e PBP2a) unlike the four native PBPs (PBP1, PBP2, PBP3, PBP4) of $S$. aureus, has remarkably reduced binding affinities to $\beta$-lactam antibiotics.Hospital acquired MRSA (HA-MRSA) are more likely to have SCCmec subtype II \& III (large molecules, more likely to also contain multidrug resistance genes) while community acquired MRSA (CA-MRSA) have subtype IV \& V (much smaller molecules, only carry $m e c \mathrm{~A}$ gene). CA-MRSA often produces a cytotoxin, Panton-Valentine leukocidin (PVL) (Lakhundi S. et al., 2018).

This study was conducted to know the prevalence and susceptibility pattern of MRSA at a tertiary care hospital. The data thus obtained would be helpful in surveillance, control and formulation of rational antibiotic policies for hospital and community acquired MRSA.

\section{Materials and Methods}

The study was conducted at a tertiary care hospital with 900 beds located in Northern India, from January 2018 to June 2019.The study population comprised of samples obtained from patients of all age and sex who were attending the hospital's out-patient department or were admitted in the hospital and showed signs \& symptoms of infection. Relevant data about the patient's illness was obtained from hospital records. 


\section{Sample collection and laboratory analysis}

The isolates of the Staphylococcus aureus for the study were obtained from clinical samples comprising of blood, urine, pus, sputum, pleural fluid, throat swab, high vaginal swab, cerebrospinal fluid, endotracheal secretions, catheter tip and other body fluids, that were received in the bacteriology section of our departmental laboratory. Preliminary identification of bacterial isolate was done by studying colony morphology, hemolysis on blood agar plate, color changes on differential media and enzymatic activity using catalase and coagulase test. Following which, Gram staining was performed for identifying Gram Positive cocci. Also, isolated colonies were sub-cultured onto nutrient agar for biochemical testing and demonstration of any pigment production (Mackie \& McCartney 2006).

MRSA identification was done by cefoxitin disk diffusion method. Inhibition zone size of $\geq 22 \mathrm{~mm}$ using cefoxitin $(30 \mu \mathrm{g})$ disk was taken as sensitive (MSSA) and zone size of $\leq 21 \mathrm{~mm}$ was taken to be Methicillin Resistant Staphylococcus aureus (MRSA) as per Clinical and Laboratory Standards Institute (CLSI) recommendations (CLSI 2018).

\section{Antibiotic susceptibility testing}

Kirby Bauer Disc Diffusion method for antibiotic susceptibility testing was performed for all the bacterial isolates according to The Clinical and Laboratory Standards Institute (CLSI) guidelines using Mueller -Hinton's Agar standard media. Commercially prepared antimicrobial discs (Hi Media Laboratories, Pvt. Limited, India) of $6 \mathrm{~mm}$ diameter were used for testing. All Staphylococcus aureus isolates were subjected to antibiotic susceptibility testing with following antibiotics- Penicillin (10 Unit), Cefoxitin (30 $\mu \mathrm{g})$, Vancomycin $(30 \mu \mathrm{g})$, Linezolid $(30 \mu \mathrm{g})$,
Teicoplanin $(30 \mu \mathrm{g})$, Amoxyclav $(30 \mu \mathrm{g})$, Clindamycin $(2 \mu \mathrm{g})$, Erythromycin $(15 \mu \mathrm{g})$, Levofloxacin $(5 \mu \mathrm{g})$, Ciprofloxacin $(5 \mu \mathrm{g})$ and Gentamicin $(10 \mu \mathrm{g})$. The Antibiotic susceptibility results were interpreted as either Sensitive, Intermediate or Resistant to an antimicrobial agent based on CLSI interpretation guidelines 2018 (CLSI 2018).

\section{Results and Discussion}

Out of the total of 158 Staphylococcus aureus isolates, $59 \quad(37.34 \%)$ were Methicillin resistant staphylococcus aureus (MRSA) and the remaining $99(62.66 \%)$ isolates were Methicillin sensitive Staphylococcus aureus (MSSA). Table 1

The MRSA isolates were further classified into hospital acquired MRSA (HA-MRSA) and community acquired MRSA (CA-MRSA). Hospital acquired MRSA infection was defined as occurring in a patient whose MRSA isolate was cultured more than 48 hours after admission to the hospital or who had a history of hospitalization, surgery, dialysis or residence in a long term health care facility within 6 months prior to the culture date or who had a indwelling intravenous line, catheter or any other percutaneous medical device present at the time the culture was taken.

Patients with none of the above conditions were classified as having community acquired MRSA infection. In our study $28(47.46 \%)$ of the MRSA isolates were hospital acquired MRSA while $31(52.54 \%)$ were community acquired MRSA. Table 2.

Sample wise distribution of the S.aureus isolates showed that the highest number of MRSA were isolated from pus (52.54\%) followed by blood (16.94\%), sputum $(8.47 \%)$ and urine $(8.47 \%)$. Table 3. 
All the isolates of Staphylococcus aureus were tested for antibiotic susceptibility as described under material and methods above. For urine samples Nitrofurantoin and Norfloxacin were also tested in addition to other antibiotics. The resistance pattern of MSSA and MRSA isolates against different antibiotics is depicted in table 4. Methicillin Sensitive Staphylococcus aureus showed $100 \%$ sensitivity to Vancomycin, Teicoplanin, Linezolid. Least sensitivity of MSSA was noted for penicillin (42.4\%) followed by Ciprofloxacin (47.5\%) and Erythromycin (67.7\%). All MSSA isolate from urine were sensitive to Nitrofurantoin. Sensitivity to Norfloxacin for urine samples was 70\%. There was no resistance of MSSA to Vancomycin, Linezolid, Teicoplanin.

Methicillin Resistant Staphylococcus aureus (MRSA) showed $100 \%$ sensitivity to Vancomycin and Teicoplanin, followed by Linezolid (98.3\%) and Amikacin (62.7\%). Isolates from urine samples showed $60 \%$ sensitivity to Nitrofurantoin and 40\% sensitivity to Norfloxacin. The least sensitivity was observed for Ciprofloxacin (16.9\%) followed by Levofloxacin (23.7\%) and Erythromycin (28.8\%). There was $100 \%$ resistance to penicillin, followed by Ciprofloxacin (83.1\%), Levofloxacin (76.3\%),
Erythromycin (71.2\%) and Gentamicin $(57.6 \%)$.

The severity of infections caused by MRSA is thought to be higher than that caused by methicillin-sensitive $S$. aureus (MSSA), not because MRSA strains are in general more virulent, but because they offer fewer treatment options. Mounting evidence suggests that MRSA infections lead to a longer stay in hospitals, which in turn leads to higher costs (Filice GA et al., 2010). The present study included a total of 158 isolates of Staphylococcus aureus. We identified 59 (37.34\%) of these as MRSA and $99(62.66 \%)$ as MSSA. A study published in 2011 by Indian Network for Surveillance of Antimicrobial Resistance (INSAR) group, was conducted in 15 Indian tertiary care centers and reported a similar prevalence of MRSA at 40\% (Joshi S. et al., 2013). Another study from northern India conducted by Shetty $\mathrm{J} e t$ al., in 2017 also reported a MRSA identification rate of $36.9 \%$ (Shetty S. et al., 2017). Arora $S$ et.al (Arora $S$ et al., 2010), in their study from Punjab reported a slightly higher prevalence of $46 \%$ while the prevalence was $38.4 \%$ in a study by Tiwari HK from Uttar Pradesh (Tiwari et al., 2008).

Table.1 Distribution of MRSA and MSSA among Staphylococcus aureus isolates

\begin{tabular}{|l|c|c|}
\hline Methicillin Susceptibility & Number (n) & Percentage (\%) \\
\hline MSSA & 99 & 62.66 \\
\hline MRSA & 59 & 37.34 \\
\hline Total & 158 & 100 \\
\hline
\end{tabular}

Table.2 Distribution of Hospital acquired and Community acquired MRSA

\begin{tabular}{|l|c|c|}
\hline & Number (n) & Percentage (\%) \\
\hline Community Acquired MRSA (CA-MRSA) & 31 & 52.54 \\
\hline Hospital Acquired MRSA (HA-MRSA) & 28 & 47.46 \\
\hline Total & 59 & 100 \\
\hline
\end{tabular}


Table.3 Sample wise distribution of MRSA

\begin{tabular}{|l|c|c|}
\hline Sample & Number (n=59) & Percentage (\%) \\
\hline Pus & 31 & 52.54 \\
\hline Blood & 10 & 16.94 \\
\hline Sputum & 5 & 8.47 \\
\hline Urine & 5 & 8.47 \\
\hline Throat/Nasal Swab & 3 & 5.08 \\
\hline Drain tip & 3 & 5.08 \\
\hline Vaginal Swab & 2 & 3.38 \\
\hline
\end{tabular}

Table.4 Antibiogram of Staphylococcus aureus (MSSA and MRSA)

\begin{tabular}{|l|c|c|c|c|c|c|}
\hline \multirow{2}{*}{ Antibiotics } & $\begin{array}{c}\text { Staphylococcus aureus } \\
\text { Resistance Pattern } \\
\text { (Total n= 158) }\end{array}$ & \multicolumn{2}{|c|}{$\begin{array}{c}\text { MSSA Resistance } \\
\text { Pattern }\end{array}$} & \multicolumn{2}{c|}{$\begin{array}{c}\text { MRSA Resistance } \\
\text { Pattern }\end{array}$} \\
\cline { 2 - 7 } & $\begin{array}{c}\text { Number } \\
(\mathrm{n}=158)\end{array}$ & Percentage (\%) & $\begin{array}{c}\text { Number } \\
(\mathrm{n}=99)\end{array}$ & $\begin{array}{c}\text { Percentage } \\
(\%)\end{array}$ & $\begin{array}{c}\text { Number } \\
(\mathrm{n}=59)\end{array}$ & $\begin{array}{c}\text { Percentage } \\
(\%)\end{array}$ \\
\hline Penicillin G & 116 & 73.4 & 57 & 57.6 & 59 & 100 \\
\hline Cefoxitin & 60 & 38 & 2 & 2.0 & 58 & 98.3 \\
\hline Amikacin & 33 & 20.9 & 11 & 11.1 & 22 & 37.3 \\
\hline Gentamicin & 51 & 32.2 & 17 & 17.2 & 34 & 57.6 \\
\hline Erythromycin & 74 & 46.8 & 32 & 32.3 & 42 & 71.2 \\
\hline Clindamycin & 43 & 27.2 & 19 & 19.2 & 24 & 40.7 \\
\hline Ciprofloxacin & 101 & 63.9 & 52 & 52.5 & 49 & 83.1 \\
\hline Levofloxacin & 83 & 52.5 & 38 & 38.4 & 45 & 76.3 \\
\hline Vancomycin & 0 & 0 & 0 & 0 & 0 & 0 \\
\hline Teicoplanin & 0 & 0 & 0 & 0 & 0 & 0 \\
\hline Linezolid & 1 & 0.6 & 0 & 0 & 1 & 1.7 \\
\hline Nitrofurantoin (15) & 2 & 13.3 & $0(\mathrm{n}=10)$ & 0 & $2(\mathrm{n}=5)$ & 40 \\
\hline Norfloxacin (15) & 6 & 40 & $3(\mathrm{n}=10)$ & 30 & $3(\mathrm{n}=5)$ & 60 \\
\hline
\end{tabular}

According to a recent study, the frequency of $45 \%$ of $S$. aureus clinical isolates being methicillin-resistant in India is similar to what has been reported in the rest of the Asian countries (41.9\% in Pakistan, 45.8\% in China, 41\% in Japan, 35.3\% in Singapore and 55.9\% in Taiwan), except Hong Kong, Indonesia (28\% each) and South Korea (>70\%) (Chen CJ et al., 2014). The differences in prevalence rates of MRSA reported from various centers might be due to several factors like different sample sizes and study population, difference in healthcare facilities, pattern of antibiotic usage and antibiotic policies and varying infection control practices from hospital to hospital. In our study 28 (47.46\%) of the MRSA isolates were hospital acquired while 31 (52.54\%) were community acquired MRSA. Many recent studies from Asia and also from western countries have shown a declining trend in the proportion of HAMRSA. The change in MRSA strains, owing to the entry of CA-MRSA strains into hospitals, has been proposed as a possible 
explanation. CA-MRSA isolates are now being increasingly reported from India. D' Souza et al., studied 412 confirmed cases of MRSA and found that $4 \%$ were true CAMRSA which was similar to our study (D’Souza N 2010).

Among all antibiotic classes, glycopeptides emerged as the most effective class of antibiotic against MRSA. All MRSA isolates showed $100 \%$ sensitivity to Vancomycin and Teicoplanin, followed by Linezolid (98.3\%) and Amikacin (62.7\%). The epidemiology of MRSA is constantly changing, with novel MRSA clones appearing in different geographical regions. Accurate and early laboratory detection of MRSA is important for institution of appropriate antibiotic treatment and also for the prevention of the spread of infection to other patients and health care personnel. We hope that this study will serve as a useful reference for clinical microbiologists, physicians and other researchers interested in the study of epidemiological characteristics and antibiotic susceptibility pattern of MRSA.

\section{References}

Arora S, et al., Prevalence of Methicillinresistant Staphylococcus aureus (MRSA) in a tertiary care hospital in northern India. J Lab Physicians, 2010; 2:78-81.

Chen CJ et al., New epidemiology of Staphylococcus aureus in Asia. ClinMicrobiol Infect, 2014; 20:60523.

Clinical and Laboratory Standards Institute (CLSI)2018. Performance standards for antimicrobial susceptibility testing; 28th Edition. CLSI Supplement M200. DeresinskiS.

Methicillin-resistant Staphylococcus aureus: an evolutionary, epidemiologic, and therapeutic odyssey. Clin Infect Dis, 2005; 40(4): 562-73.

D'Souza N, et al., Molecular characterization of Methicillin-resistant Staphylococcus aureus with emergence of epidemic clones of sequence type (ST)22 and ST 772 in Mumbai, India. J Clin Microbiol., 2010;48:1806-11.

Filice GA,et al., Excess costs and utilization associated with methicillin resistance for patients with Staphylococcus aureus infection. Infect Control Hosp Epidemiol, 2010; 31:365-73.

Gopalakrishnan $\mathrm{R}$, et al., Changing trends in antimicrobial susceptibility and hospital acquired infections over an 8year period in a tertiary care hospital in relation to introduction of an infection control programme. J Assoc Physicians India, 2010; 58: 25-31.

Joshi S, et al., Methicillin resistant Staphylococcus aureus (MRSA) in India: Prevalence and susceptibility pattern. Indian J Med Res., 2013; 137(2): 363-9.

Lakhundi S. Methicillin resistant Staphylococcus aureus: molecular characterization, evolution, and epidemiology. Clin Microbiol Rev, 2018;31(4):1-32.

Mackie \& McCartney practical Medical Microbiology 2006, Tests for the identification of Bacteria, $14^{\text {th }}$ edition, Delhi: Elsevier publication, 131-150.

Patel AK, et al., Time trends in the epidemiology of microbial infections at a tertiary care centre in west India over last 5 years. J Assoc Physicians India 2010; 58: 37-40.

Robert C. Moellering. MRSA: the first half century. Journal of Antimicrobial Chemotherapy,2012; 67(1): 4-11.

Shetty $\mathrm{J}$ et al., Prevalence of constitutive and inducible clindamycin resistance among clinical isolates of Staphylococcus aureus in a tertiary 
care institute in North India. Int J Res Med Sci., 2017; 5(7):3120-25.

Tiwari HK, et al., 2008. High prevalence of multidrug-resistant MRSA in a tertiary care hospital of northern India.
Infection and Drug Resistance,2008; $1: 57-61$.

WHO 2014. Antimicrobial Resistance: Global Report on Surveillance.

\section{How to cite this article:}

Ayesha Nazar, Yusuf Imran, Vichal Rastogi and Parul Singhal 2019. Prevalence and Antibiotic Susceptibility Pattern of Methicillin Resistant Staphylococcus aureus (MRSA) at a Tertiary Care Hospital from Northern India. Int.J.Curr.Microbiol.App.Sci. 8(09): 2352-2358.

doi: https://doi.org/10.20546/ijcmas.2019.809.271 